\title{
A Multi-drug resistance pattern of a Leclercia adecarboxylata strain isolated from a urinary tract infection of a patient at China-Guinea friendship hospital of Kipé/Conakry
}

\author{
Abdoulaye MAKANERA ${ }^{1,2^{*}}$, Mariam CONDE ${ }^{1}$, Mamadou Alpha DIALLO ${ }^{1}$, \\ Mariama CONDE ${ }^{1}$, Daouda CAMARA ${ }^{1}$, Tiguidanké DIAKITE ${ }^{1}$ and \\ Alpha Oumar BARRY ${ }^{1}$ \\ ${ }^{1}$ Laboratoire biomédical, Hôpital de l'Amitié Sino-Guinéenne (HASIGUI), Cité des Médecins Kipé-Ratoma \\ 030 BP: 710, Conakry, République de Guinée. \\ ${ }^{2}$ Chaire de Bactériologie-Virologie, Département Sciences Fondamentales, FMPOS, Université Gamal Abdel \\ Nasser Conakry, République de Guinée. \\ *Corresponding author; E-mail: abdmak@yahoo.fr; Tél. +224 664309523/+224 621015770;
}

\begin{abstract}
Leclercia adecarboxylata $(L A D)$ is a member of Enterobacteriaceae family that is usually reported as an opportunistic human pathogen. A few reports have described resistant strains in the literature. The aim of this paper was to describe the antimicrobial resistance pattern of a $L A D$ strain isolated from a urinary tract infection in a 39-year-old immunocompetent man. The bacterial identification and antibiotic sensitivity tests were performed on Vitek 2 Compact 15. The results revealed the presence of $L A D$ with a particular multidrug resistance pattern. It was sensitive only to imipenem $(=1 \mu \mathrm{g} / \mathrm{ml})$, and totally resistant to association of trimethoprim/sulfamethoxazole $(\geq 320 \mu \mathrm{g} / \mathrm{ml})$, ticarcillin $(\geq 128 \mu \mathrm{g} / \mathrm{ml})$, nitrofurantoin $(=128 \mu \mathrm{g} / \mathrm{ml})$, cefalothin ( $\geq 64 \mu \mathrm{g} / \mathrm{ml})$, cefoxitin $(\geq 64 \mu \mathrm{g} / \mathrm{ml})$, cefotaxime $(\geq 64 \mu \mathrm{g} / \mathrm{ml})$, ceftazidime $(\geq 64 \mu \mathrm{g} / \mathrm{ml})$, amikacin $(\geq 64 \mu \mathrm{g} / \mathrm{ml})$, ampicillin $(\geq 32 \mu \mathrm{g} / \mathrm{ml})$, nalidxic acid $(\geq 32 \mu \mathrm{g} / \mathrm{ml})$, and a combination of amoxicillin/clavulanic acid $(\geq 32 \mu \mathrm{g} / \mathrm{ml})$, gentamicin $(\geq 16 \mu \mathrm{g} / \mathrm{ml})$, tobramycin $(\geq 16 \mu \mathrm{g} / \mathrm{ml})$, ofloxacin $(\geq 8 \mu \mathrm{g} / \mathrm{ml})$, and ciprofloxacin $(\geq 4 \mu \mathrm{g} / \mathrm{ml})$. It showed the intermediate sensitivity to the association of piperacillin/tazobactam $(=64 \mu \mathrm{g} / \mathrm{ml})$, and ertapenem $(=4 \mu \mathrm{g} / \mathrm{ml})$. The findings showed that this isolate of $L A D$ had a multidrug resistance pattern to almost all the antibiotics tested (except imipenem). This suggests that $L A D$ could be considered as an emergent bacterial pathogen capable of causing infections in human and carrying multidrug resistance pattern to numerous antibiotic families in Guinea. (C) 2018 International Formulae Group. All rights reserved.
\end{abstract}

Keywords: Leclercia adecarboxylata, multi-drug, resistance, Kipé/Conakry

\section{INTRODUCTION}

Leclercia adecarboxylata $(L A D)$ is an opportunistic human pathogen which was designated initially as "Enteric group 41" or Escherichia adecarboxylata in 1962 by Leclerc (Kashani et al., 2014; Grantham et al., 2015). Advances of diagnostic technology particularly in the molecular biology fields allowed to separate Escherichia adecarboxylata from the "Enterobacter agglomerans" complex and to name it as $L A D$ (De Mauri et al., 2013; Kashani et al., 2014; Hurley et al., 2015).

$L A D$ is a motile Gram negative rod belonging to the Enterobacteriacea family. 
The species are facultative anaerobic bacteria, oxydase negative, peritrichously flagellated bacilli (Stock et al., 2004). This organism shares many biomedical features with Escherichia coli.

Leclerc et al. first reported the isolation of $L A D$ from drinking water in 1962 (Grantham et al., 2015). Further reports showed that it is widely distributed in nature (food, water), a part of normal flora in the gut of animals and in the stools of humans (Keren et al., 2014; Tam and Nay, 2012; Stock et al., 2004). It has also been reported in various clinical samples including blood, urine, sputum, wounds pus, synovial fluid, peritoneal fluid, gallbladder tissue, and cardiac valve tissue (Forrester et al., 2012; Tam and Nayaka, 2012; Dalamaga et al., 2015; Jean et al., 2016). $L A D$ is capable to cause infection in immunocompetent human (Hess et al., 2008).

The aim of this study was to report the first isolation of $L A D$ in Guinea from a urinary tract infection in humans and determine the multi-drug resistance pattern of this emerging bacterial pathogen.

\section{MATERIALS AND METHODS}

A 39 year-old-male patient was admitted for hypertension in the Neurology department of China-Guinea Friendship hospital of Kipé (Conakry/Guinea) on October $13^{\text {th }}$ 2016. The patient was suffering from urinary tract infection. Some biological parameters of the patient were investigated. The HIV immunoserological test was performed using both HIV Combo (Alere Medical Co., Ltd, Mtsudo-shi, Japan) and Enzyme Linked Immunosorbent Assay (ELISA) on miniVidas (bioMérieux, Marcy l'Etoile, France).

The urine cultures were done on agar plates of different media: Nutrient agar
(Liofilchem, Italy), blood agar (Liofilchem, Italy), and CLED (bioMérieux, Narcy, l'Etoile, France). After 24 hours incubation cultures yielded pure and large colonies of gram negative bacilii. Gram stained smears were observed on photonic binocular microscopy (Microscope XS-213, China). The bacterial identification and antibiotic susceptibility tests (antibiogram) as well as the determination of minimal inhibition concentrations (MIC) were processed on Vitek 2 Compact 15 system (bioMérieux, Narcy, l'Etoile, France). The Vitek 2 GN card (bioMérieux, Narcy, l'Etoile, France) was used for bacterial identification and the Vitek 2 N 233 card (BioMérieux, Narcy, l'Etoile, France) was used for antibiogram and determination of MIC, according to manufacturer's instructions.

\section{RESULTS}

Our results showed that the patient was HIV negative. The bacterial isolate was identified by Vitek 2 Compact as Leclercia adecarboxylata. Culture on nutrient agar plate is shown in Figure 1.

The results of antibiotic sensitivity tests of $L A D$ strain are shown in Table 1.

This isolate was resistant to nearly all antibiotic families tested. In fact, it was resistant to all tested quinolones, aminoglycosides, folate pathway inhibitors (sulfamides) and nitrofurantoin. For betalactams, this strain was resistant to all cephalosporins tested and nearly to all penicillins, except the association piperacillin/Tazobactam, to which it showed intermediate sensitivity. It showed total sensitivity to imipenem (carbapenem) and the intermediate sensitivity to ertapenem. 
Table 1: Antibiotic sensitivity of Leclercia adecarboxylata isolate with the minimal inhibition concentrations (MIC).

\begin{tabular}{lcclcc}
\hline Antibiotics & Sensitivity & $\begin{array}{c}\text { MIC } \\
(\boldsymbol{\mu g} / \mathbf{m l})\end{array}$ & Antibiotics & Sensitivity & $\begin{array}{c}\text { MIC } \\
(\boldsymbol{\mu g} / \mathbf{m l})\end{array}$ \\
\hline Ampicillin & $\mathrm{R}$ & $\geq 32$ & Imipenem & $\mathrm{S}$ & 1 \\
Amoxicillin/Cla-vulanic & $\mathrm{R}$ & $\geq 32$ & Amikacin & $\mathrm{R}$ & $\geq 64$ \\
acid & $\mathrm{R}$ & $\geq 128$ & Gentamicin & $\mathrm{R}$ & $\geq 16$ \\
Ticarcillin & $\mathrm{I}$ & 64 & Tobramycin & $\mathrm{R}$ & $\geq 16$ \\
Piperacillin/Tazo-bactam & $\mathrm{R}$ & $\geq 64$ & Nalidixic acid & $\mathrm{R}$ & $\geq 32$ \\
Cefalotin & $\mathrm{R}$ & $\geq 64$ & Ciprofloxacin & $\mathrm{R}$ & $\geq 4$ \\
Cefoxitin & $\mathrm{R}$ & $\geq 64$ & Ofloxacin & $\mathrm{R}$ & $\geq 8$ \\
Cefotaxime & $\mathrm{R}$ & $\geq 64$ & Nitrofurantoin & $\mathrm{R}$ & $\geq 128$ \\
Ceftazidime & $\mathrm{I}$ & 4 & Trimethoprim/Sulfamethoxazole & $\mathrm{R}$ & $\geq 320$ \\
Ertapenem & &
\end{tabular}

MIC: minimal inhibition concentration; I: intermediate, R: resistant; S: sensitive;

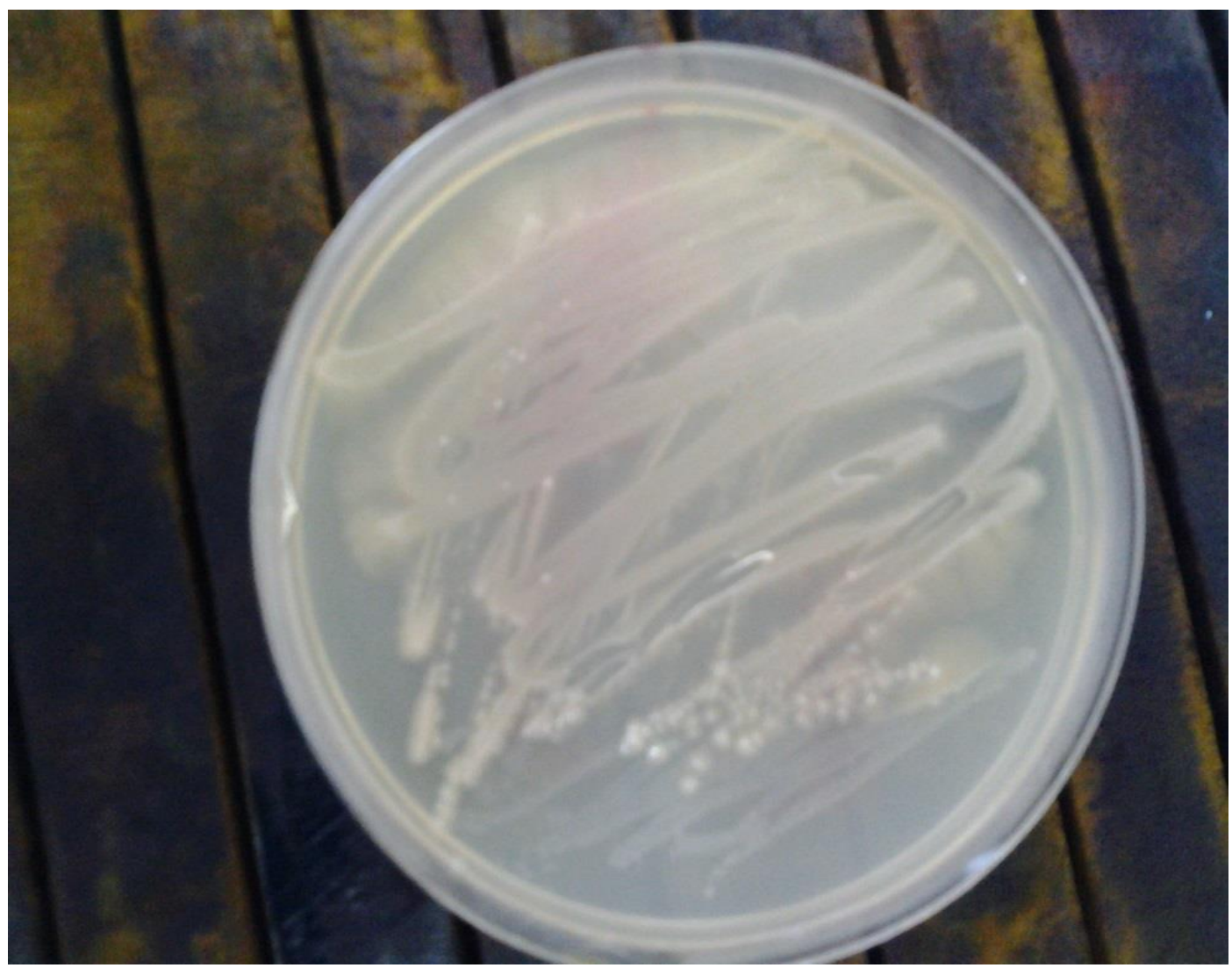

Figure 1: Colonies of Leclercia decrboxylta isolated on nutrient plate agar medium (cultures were incubated for 24 hours at $37^{\circ} \mathrm{C}$ ). 


\section{DISCUSSION}

Numerous reports have revealed that $L A D$ is widely distributed in nature (food, water), and is a part of normal flora in the gut of animals and in the stools of humans (Keren et al., 2014; Tam et Nay, 2012; Stock et al., 2004). It has also been reported in the various clinical samples including blood, urine, sputum, wounds pus, synovial fluid, peritoneal fluid, gallbladder tissue, sublingual splinter, and cardiac valve tissue (Forrester et al., 2012; Tam and Nayak, 2012; Dalamaga et al., 2015, Jean et al., 2016). Some authors have reported that the epidemiological significance of Leclercia. adecarboxylata is not clear (De Mauri et al., 2013). The paucity of reports of human infection in the past may reflect misdiagnosis, as the organism shares many biochemical features with Escherichia coli, rather than a true incidence ef human infection. Some reports showed that $L A D$ has been isolated from patients with mixed microbial infection (Saccani et al., 2017), which raises questions concerning the organism's role in some of these infections. Moreover, some investigators reported that $L A D$ has been isolated from polymicrobial cultures from immunocompetent patients and as a pure culture from immunocompromised patients, which suggests the dependence of this bacterium on co-flora to cause a disease (Tam and Nayak, 2012).

In the present study, we isolated $L A D$ in a mono-microbial urinary tract infection in immunocompetent man instead of a polymicrobial infection. This is different from others observations (Saccani et al., 2017). Our data suggest that this isolate could be considered as an emergent pathogen. It is therefore of interest in that it might be considered to our knowledge as the first report in the literature describing the isolation of this organism in a pure culture of a urinary tract infection of an immunocompetent man. Numerous authors (Tam et Nayak, 2012; Hess et al., 2008; Grantham et al., 2015) have reported isolation of LAD in blood cultures of immunocompetent patients (Mazzariol et al., 2003), and in an abscess (Hess et al., 2008).
There are few published data in the literature dealing with antimicrobial susceptibility patterns of $L A D$. In all cases, only a small number of antibiotics (up to a maximum of 13) were tested. In addition, only a few data are available on the natural antimicrobial susceptibilities of $L A D$. It is generally sensitive to most antibiotics and only a few reports revealed some resistant isolates (Dalamaga et al., 2009; Tam and Nayak, 2012; Shin et al., 2012; Eiland et al., 2013; Anuradha, 2014; Allawh and Camp, 2015). In the present study, data on the antibiotic sensitivity tests showed that our $L A D$ isolate was sensitive only to imipenem. It was totally resistant to ampicillin, the association amoxicillin/ clavulanic acid, ticarcillin, cefalotin, cefoxitin, cefotaxime, ceftazidime, amikacin, gentamicin, tobramycin, nalidixic acid, ciprofloxacin, ofloxacin, nitrofurantoin and the trimethoprim/sulfamethoxazole. Finally, it showed the intermediate sensitivity to ertapenem and the association of piperacillin/tazobactam (Table 1).

The antimicrobial sensitivity tests revealed that this strain was resistant to nearly all antibiotic families tested. In fact, it was resistant to all tested quinolones, aminoglycosides, folate pathway inhibitors (sulfamides) and nitrofurantoin. For betalactams, this strain was resistant to all cephalosporins tested and to all penicillins, except the association piperacillin/tazobactam, to which it showed intermediate sensitivity. The only one total sensitivity was shown to imipenem (carbapenem) while intermediate sensitivity was shown to ertapenem. These data are very different from those of numerous authors Kashani et al., 2014; Hurley et al., 2015; Saccani et al. 2017). Indeed, in Germany, Stock and his colleagues conducted the first large study on natural antimicrobial susceptibility patterns and biochemical profiles of 101 LAD strains (Stock et al., 2004). Their data revealed that most of the strains tested were sensitive to all tested antimicrobial agents, apart from benzylpenicillin. Anuradha (2014) in his reports on LAD strains isolated in two case 
reports showed that his strains were sensitive to nearly all antibiotics tested (Anuradha, 2014). Also, Kashani et al. (2014) reports on antimicrobial susceptibility tests of $L A D$ strains showed that these were susceptible to all the antibiotics tested (ampicillin, cefazolin, ciprofloxacin, gentamicin, imipenem, levofloxacin, tobramycin, trimethoprim /sulfamethoxazole. More recently, Saccani et al. (2017) showed that Leclercia adecarboxylata has a broad sensitivity to the majority of antibiotics (beta-lactams, quinolones, azithromycin, aminoglycosides, and tetracyclines). Mazzariol et al. (2003) have previously reported that their strain was sensitive to numerous antibiotics including amoxicillin/clavulanic acid, cefoxitin, gentamicin, ciprofloxacin (Mazzariol et al., 2003). Their isolate was susceptible to imipenem but resistant to cefotaxime and ceftazidime. This data is then very similar to ours. Shin et al. (2012) showed their LAD isolate was resistant only to carbapenems and quinolones, but it was sensitive to aminoglycosides (amikacin, gentamicin, tobramycin), most beta-lactactams, including broad spectrum cephalosporins (cefotaxime and cefixime) and trimethoprime/sulfamethoxazole.

Considering these and other authors published data, there is evidence that Leclercia adecarboxylata strains are usually sensitive to numerous antimicrobial agents. Hurley et al. (2015) have reported a pan-drug sensitive isolate of $L A D$, except for intermediate sensitivity for piperacilline/tazobactam.. In contrast, our data showed that the isolate of Leclercia adecarboxylata described in the present study was resistant to nearly all the antibiotics tested $(15 / 16=93,75 \%)$ except to imipenem. To our knowledge, this is the first report in the literature that described a Leclercia adecarboxylata strain isolated in pure monomicrobial culture from urinary tract infection in immunocompetent patient, and which was resistant to $93,75 \%$ of antibiotics tested belonging to different families. In fact, this multidrug-resistance pattern suggest, an obvious therapeutic difficulty for treatment of
$L A D$ infections. Then, $L A D$ seems becoming a microbial pathogen like other classical microbes in the world (Makanéra et al., 2003; Okorundu et al., 2013; Koanga Mogtomo et al., 2016; Abba et al., 2017; Makanéra et al., 2017; Tchapdie Ngassam et al., 2017).

Our findings revealed that $L A D$ could be considered as an emerging bacterial threaten pathogen to human health, because of its multidrug-resistance pattern which includes numerous antibiotic families.

\section{COMPETING INTERESTS}

The authors declare that they have no competing interests.

\section{AUTHORS' CONTRIBUTIONS}

TD and AOB: collected urine samples; AM, MC, MC, DC, MAD: contributed in the laboratory analyses; AM and MLK contributed in the collection of bibliography and manuscript drafting. AM: the main investigator did the conception of the protocol and manuscript writing.

\section{ACKNOWLEDGEMENTS}

This work was financed by grants from The Health Department of Republic of Guinea and The Popular Republic of China.

\section{REFERENCES}

Abba H, Somda MK, Antipas BB, Barro N, Traore AS. 2017. Prévalence et susceptibilité aux antibiotiques des souches de Salmonella spp. non typhiques isolées de la viande de poulets au Tchad. Int. J. Biol. Chem. Sci., 11(1): 107-117. DOI: http://dx.doi.org/10.4314/ijbcs.v11i1.9

Allawh R, Camp BJ. 2015. Isolation of Leclercia adecarboxylata from a patient with a subungual splinter. Dermatol Online J., 21(8). pii: 13030/qt2hb6x3c9.

Anuradha M. 2014. Leclercia adecarboxylata Isolation: Case Reports and Review. $J$ Clin Diagnostic Res., 8(12): DD03-DD04

Dalamaga M, Pantelaki M, Karmaniolas K, Daskalopoulou K, Migdalis I. 2009. Isolation of Leclercia adecarboxylata 
from blood and burn wound after a hydrofluoric acid chemical injury. Burns., 35(3): 443-445.

DOI:10.1016/j.burns.2008.01.02.

De Mauri A, Chiarinotti D, Andreoni S, Molinari GL, Conti N, De Leo M. 2013. Leclercia adecarboxylata and catheterrelated bacteraemia: review of the literature and outcome with regard to catheters and patients. J. Medical. Microbiol., 62: 1620-1623. DOI: 10.1099/jmm.0.059535-0

Eiland EH, Siddiqui H, Goode AM, Leeth SD. 2013. Pneumonia due to multidrugresistant Leclercia adecarboxylata. American Journal of Health-System Pharmacy., 70(11): $940 . \quad$ DOI: https://doi.org/10.2146/ajhp120525

Forrester JD, Adams J, Sawyer RG. 2012. Leclercia adecarboxylata bacteremia in a trauma patient: Case report and review of literature. Surg Infect (Larchmt), 13(1):63-66. DOI: 10.1089/sur.2010.093

Grantham WJ, Funk SS, Schoenecker JG. 2015. Leclercia adecarboxylata Musculoskeletal Infection in an Immune Competent Pediatric Patient: an Emerging pathogen? Hindawi Publishing Corporation Case Reports in Orthopedics Volume 2015, 3 pages. DOI: $10.1155 / 2015 / 160473$

Hess B, Burchett A, Huntington MK. 2008 "Leclercia adecarboxylata in an immunocompetent patient. J. Med. Microbiol., 57(7): 896-898. DOI: 10.1099/jmm.0.47673-0.

Hurley EH, Cohen E, Katarincic JA, Ohnmacht RK. 2015. Leclercia Adecarboxylata Infection in an Immunocompetent Child. Rhode Island Medical Journal.: 41-44 http://www.rimed.org/rimedicaljournal 2015/09/2015-09-41-case-hurley.pdf Jean S-S, Lee W-S, Bai K-J, Lam C, Hsu CW, Chen R-J, Hsueh P-R. 2016. Leclercia adecarboxylata bacteremia in a patient with long-term use of nonsteroidal antiinflammatory drugs. J. Microbiol., Immunol. Infect., 49: 452-454. https://doi.org/10.1016/j.jmii.2013.09.00 4

Kashani A, Chitsazan M, Che K, Roger C. Garrison RC. 2014. Leclercia adecarboxylata Bacteremia in a Patient with Ulcerative colitis. Case Reports in Gastrointestinal Medicine, 4 pages. http://dx.doi.org/101155/2014/457687

Keren Y, Keshet D, Eidelman M, Geffen Y, Raz-Pasteur A, Hussein K. 2014. Is Leclercia adecarboxylata a new and unfamiliar marine pathogen? J. Clin. Microbiol., $\quad \mathbf{5 2}$ : 1775-1776. DOI: $10.1128 /$ JCM.03239-13

Koanga MML, Ngo NA , Longang AM, Kojom FLP, Embolo E, Kom B, Wandja PV, Tchamdjeu F, Ngono Ngane AR. 2016. Prévalence des germes impliqués dans les infections vaginales chez les femmes camerounaises et facteurs de risqué. Int. J. Biol. Chem. Sci., 10(1): 255-268. DOI : http://dx.doi.org/10.4314/ijbcs.v10i1.20

Makanéra A, Condé M, Diallo MA. 2017. Profil de sensibilité des souches de Sphingomonas paucimobilis isolées de diverses secrétions biologiques à l'Hôpital de l'Amitié Sino-guinéenne kipé/Conakry (Guinée). REV. CAMES Science de la Vie, de la Terre et Agronomie, $\quad \mathbf{0 5}(01): \quad$ 14-20. publication.lecames.org/index.php/svt/ar ticle/view/95

Makanéra A, Arlet G, Gautier V, Manai M. 2003. Molecular Epidemiology and Characterization of Plasmid-Encoded $\beta$ Lactamases Produced by Tunisian Clinical Isolates of Salmonella enterica Serotype Mbandaka Resistant to BroadSpectrum Cephalosporins. J. Clin. 
Microbiol., $\quad$ 41(7): 2940-2945. DOI:10.1128/JCM.41.7.2940-2945.2003

Mazzariol A, Zuliani J, Fontana R, Cornaglia. 2003. Isolation from blood culture of a Leclercia adecarboxylata strain producing an SHV-12 extended spectrum beta-lactamase. J. Clin. Microbiol., 41: 1738-9. DOI:10.1128/JCM.41.4.1738-1739.2003

Okorondu SI, Akujobi CO, Nnadi CB, Anyado-Nwadike SO, Okorondu MMO. 2013. Prevalence and antibiotic sensitivity profile of urinary tract infection pathogens among pregnant and non-pregnant women. Int. J. Biol. Chem. Sci. 7(4): 1668-1677. DOI: http://dx.doi.org/10.4314/ijbcs.v7i4.22

Saccani B, Izzo I, Sasari S, Magro P, Ravizzola G, Spinetti A, Castelli F. 2017. Leclercia adecarboxylata isolation from blood cultures: an emerging pathogen in immunocompromised hosts? Infect. Dis. Trop. Med., 3(1): e-364 http ://www.infectiousjournal.com

Shin GW, You MJ, Lee HS, Lee CS. 2012. Catheter-related bacteremia caused by multidrug-resistant Leclercia adecarboxylata in a patient with breast cancer. J. Clin. Microbiol., 50(9): 31293132. DOI: $10.1128 / J C M .0094812$
Stock I, Burak S, Wiedemann B. 2004. Natural antimicrobial susceptibility patterns and biochemical profiles of Leclercia adecarboxylata strains. Clin. Microbiol. Infect., 10(8): 724-33. DOI:10.1111/j.1469-0691.2004.00892.x

Stone JP, St Denis-Katz H, Claire TempleOberle C, Mercier P, Mizzau JB, Alim P, Mitha AP. 2014. Leclercia adecarboxylata: The First Reported Infection of Cerebrospinal Fluid and a Systematic Review of the Literature. $J$. Neuroinfect. Dis., 6(3). DOI:10.4172/2314-7326.1000181

Tam V, Nayak S. 2012. Isolation of Leclercia adecarboxylata from a wound infection after exposure to hurricane-related floodwater. BMJ Case Reports, DOI:10.1136/bcr-2012-007298

Tchapdie NRF, Tantse M, Fotsing KPR, Noukela NDP, Kouamouo J, Simo LJ, Gamwo DS, Tchoukoua SH, Tamatcho KBP, Kourouma K. 2017. Multicenter study on antibiotic susceptibility/resistance trends in the western region of Cameroon. Int. J. Biol. Chem. Sci., 11(1): 131-143. DOI: http://dx.doi.org/10.4314/ijbcs.v11i1.11 